\title{
Improved ELM scaling with impurity seeding in JET
}

\author{
G P Maddison ${ }^{1}$, R V Budny ${ }^{2}$, P Dumortier ${ }^{3,8}$, S Jachmich $^{3,8}$, A Loarte $^{4}$, \\ A M Messiaen ${ }^{3,8}$, P Monier-Garbet ${ }^{5}$, M F F Nave ${ }^{6}$, J Ongena ${ }^{3,8}$, \\ J Rapp ${ }^{7,8}$, J D Strachan ${ }^{2}$ and JET EFDA contributors ${ }^{9}$ \\ ${ }^{1}$ EURATOM/UKAEA Fusion Association, Culham Science Centre, Abingdon, Oxon \\ OX14 3DB, UK \\ 2 Princeton Plasma Physics Laboratory, Princeton University, NJ 08543, USA \\ ${ }^{3}$ LPP/ERM-KMS, Association EURATOM-Belgian State, B-1000 Brussels, Belgium \\ ${ }^{4}$ EFDA-CSU, D-85748 Garching, Germany \\ ${ }^{5}$ CEA Cadarache, F-13108 St Paul lez Durance, France \\ ${ }^{6}$ CFN, Associação EURATOM-IST, 1096 Lisbon, Portugal \\ ${ }^{7}$ IPP, Forschungszentrum Jülich GmbH, EURATOM Association, D-52425 Jülich, Germany \\ E-mail: geoff.maddison@ukaea.org.uk
}

Received 28 February 2003

Published 19 August 2003

Online at stacks.iop.org/PPCF/45/1657

\begin{abstract}
Argon-seeded H-modes in low triangularity pumped-limiter-like, and medium to high triangularity divertor, configurations on JET are described, focusing upon ELM effects. Appropriate fuelling and seeding strategies in each geometry have combined good confinement with density around the Greenwald level, accompanied by ELMs shown to have explicitly Type I character. For lower triangularity, argon injection leads to a reduction in normalized ELM energy losses averaged over several fluctuations, relative to unseeded scaling at comparable frequency and confinement but lower density. This generalizes a similar result in earlier studies $[14,15]$. Optimized seeding tends always to decrease ELM frequencies too, so that the average energy efflux in ELMs is significantly diminished, down to only $\approx 10 \%$ of input power in the lowest example included. At least for lower triangularity again, electron pedestal temperature is cooled by argon, which tends also to lower peak pedestal pressure before each consequently smaller fluctuation. However, the pedestal density is raised, and at higher radiation fractions this can restore unseeded peak pedestal pressure and roughly ELM size, but still at lower frequency. The underlying physics of Type I instabilities defined by pedestal collisionality appears to prevail in all cases both with and without seeding. High performance $\mathrm{H}$-modes with both reduced core transport and moderated steady and transient power loads through added impurities therefore seem to come closer to a fully integrated scenario for $Q=10$ operation in ITER.
\end{abstract}

\footnotetext{
8 Partners in the Trilateral Euregio Cluster (TEC).

9 See appendix of paper by Pamela J 2002 19th IAEA Fusion Energy Conf. (Lyon, October 2002). 


\section{Introduction}

Latest developments of ELMy H-mode plasmas have now achieved or even slightly exceeded the global normalized properties (energy confinement $H_{98} \approx 1$, Greenwald density fraction $f_{\text {Gwd }} \approx 0.85$, pressure $\beta_{\mathrm{N}} \approx 1.8$, purity $Z_{\text {eff }} \leqslant 1.7$ ) required for $Q=10$ standard operation in ITER [1-3]. Previous difficulties with the H-mode density limit [4], whereby confinement and refuelling efficiency declined above $f_{\mathrm{Gwd}} \approx 0.7$, have been overcome in effect by extending upwards the range of Type I ELM fluctuations, which typically accompany the strongest edge pedestals and best H-mode performance [3]. At the same time, this has re-emphasized the need to mitigate their transient energy fluxes onto boundary surfaces, since these would extrapolate to severe intermittent loads in ITER liable to shorten the endurance of components and to cause contamination of the plasma [5]. One recourse being pursued is a translation to Type II ELMs, which smooth out plasma effluxes in benign, high frequency fluctuations without compromising confinement. While these have been found at strong plasma shaping in a number of tokamaks (DIII-D [6], JT-60U [7], ASDEX-U [8]), however, they have not so far emerged unequivocally in JET [3]. An alternative approach applicable at both low and high shaping, on the other hand, is seeding of the plasma edge with medium $Z$ impurities to increase dispersal of efflux power in a radiating mantle [9]. This has itself been found to be a way of achieving high density, well confined H-modes in several devices (ASDEX-U [10], JT-60U [2], JET $[1,11,12]$ ) and simultaneously has been shown to have a powerful effect on ELMs $[2,10,12-14]$. Optimizing this influence might therefore combine acceptable divertor operation with satisfactory core conditions, moving closer to a fully integrated ITER standard scenario.

In this report, we extend initial reviews $[12,14,15]$ of ELMs in seeded H-modes on JET to generalize their response over a larger database of plasmas at low and high triangularity where performance has been specifically enhanced using argon. Complementary studies injecting nitrogen are discussed elsewhere $[15,16]$. The different configurations adopted are first introduced, and the approaches developed for low and high shaping defined. Crucial modifications to Type I ELM frequencies and energy amplitudes are then described, in particular demonstrating that scalings $[17,18]$ established for unseeded plasmas are beneficially departed from in the presence of argon. Underlying changes in pedestal properties are next briefly examined. Hence the effects contributing to ELM modifications at lower triangularity are clarified. Finally, a summary is given, before considering the prospective advantages of impurity seeding for integrated states in ITER.

\section{Magnetic configurations, seeding techniques and performance effects}

A variety of different plasma configurations and seed impurity species has been investigated on JET [11, 12, 15], but here we concentrate upon argon dosing in three magnetic geometries intended to span ranges in divertor pumping and plasma shaping (see figure 1). In particular, the upper triangularity $\delta^{\mathrm{u}}$ is varied, since this is known itself strongly to affect ELM characteristics [3]. In the JET MkIIGB divertor design [18], a pumped-limiter-like arrangement was obtainable by lowering the magnetic X-point onto or just inside the dome of a dividing structure between its inboard and outboard sides, yielding the so-called 'septum' configuration, figure $1(a)$. This typically low triangularity $\left(\delta^{\mathrm{u}}=0.18\right)$ option tended to displace recycling from the close vicinity of the cryopump entrance in the divertor outboard lower corner, and in addition resulted in a lower power threshold for transition into H-mode, although the cause has not been clearly resolved [19]. For the latter reason, however, impurity seeding of H-modes in JET focused initially on septum geometry [11-13], since more radiation should 


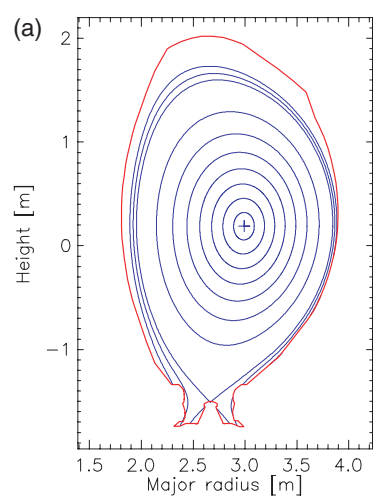

2.4-2.5T, 2.4-2.5 MA, 12-16MW $\kappa=1.65, \delta^{\mathrm{l}}=0.24, \delta^{\mathrm{u}}=0.18$

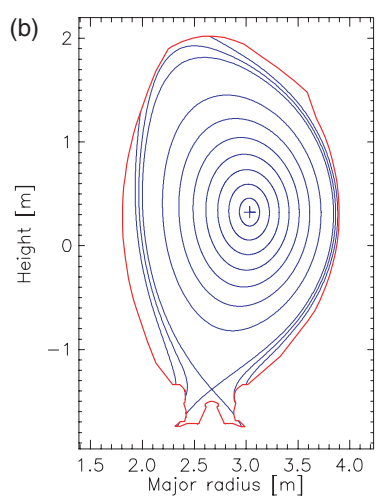

$2.4-2.5 \mathrm{~T}, 2.5 \mathrm{MA}, 13-15 \mathrm{MW}$ $\kappa=1.76, \delta^{l}=0.28, \delta^{u}=0.35$

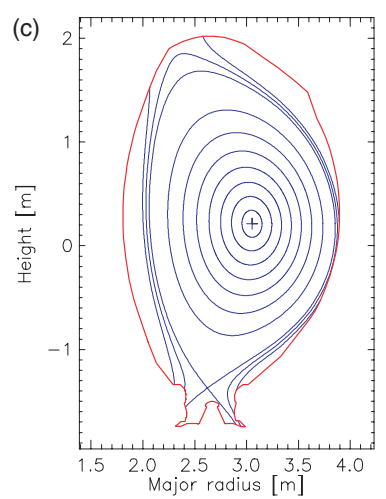

$2.4 \mathrm{~T}, 2.3-2.5 \mathrm{MA}, 14-18 \mathrm{MW}$ $\kappa=1.70, \delta^{\mathrm{l}}=0.36, \delta^{\mathrm{u}}=0.46$

Figure 1. Magnetic configurations and basic parameter ranges in JET seeding studies: (a) 'Septum'(S), low triangularity, pumped-limiter-like; (b) Vertical Targets standard divertor (VT), medium triangularity; (c) Extra High Triangularity Divertor (EHT). Note that the cryopump is located at the outboard lower corner of the MkIIGB divertor.

be tolerable without reverting to L-mode. Subsequently, a representative divertor configuration with stronger pumping and medium triangularity $\left(\delta^{\mathrm{u}}=0.35\right)$ has been addressed using the so-called 'vertical targets' (VT) case, where the magnetic strike-points are positioned on the MkIIGB side plates, figure 1(b). Finally, an adaptation of this arrangement has been made to raise the upper triangularity as far as possible within the MkIIGB design, resulting in the 'extra high triangularity' (EHT) $\left(\delta^{\mathrm{u}}=0.46\right)$ equilibrium, figure $1(c)$. Basic parameters applied in each configuration are also tabulated in figure 1 , showing particularly that relatively narrow ranges of input power have been chosen, to minimize its influence on results.

A recognized effect of trying to increase the density in H-mode at low triangularity by strong gas puffing is that confinement tends to be degraded while ELMs are compressed to smaller, but higher frequency, Type III proportions [4]. On the other hand, confinement tends to recover again, plus Type I ELMs are re-established, after puffing ends, or is greatly reduced, and a key factor noted in early JET experiments was that if seed impurities were also added, a high density tended to be retained even after the fuelling [20]. A method of optimized fuelling and radiation has correspondingly been developed, as follows. First, a strong puff of deuterium accompanied by argon seeding is applied to raise plasma density and radiation, at the expense of degraded confinement. This 'puff' phase (P) is then ended, and in the subsequent 'afterpuff' phase (AP) only very small spurts of each gas are added to maintain conditions. Such a strategy has actually led to a low $\delta$ regime combining high density $f_{\mathrm{Gwd}} \geqslant 0.9$ with recovered good confinement $H_{98} \approx 1$, while the radiated power fraction is increased to $f_{\text {rad }} \approx 0.5$ for little relative change in $Z_{\text {eff }} \approx 2.2$, all sustained for up to $\approx 12$ energy confinement times, as already reported $[1,11,12]$. Here it has been applied in both septum and vertical targets cases, as illustrated for the latter in figure 2.

At high triangularity, H-modes become more robust against gas puffing [3,21], so that good confinement can be maintained even during fuelling to higher plasma density $[1,11]$. The means of lifting the H-mode density limit encountered at lower $\delta$ remains under study [21], but for seeding experiments attention has therefore been directed to injecting argon into high $\delta$ puff stages too, again to try to maximize radiation and performance. A summary of improved conditions reached in cases for all three configurations presented is given in figure 3 , where it can be seen that seeded low $\delta$ afterpuffs and high $\delta$ puffs indeed approach the Greenwald density 

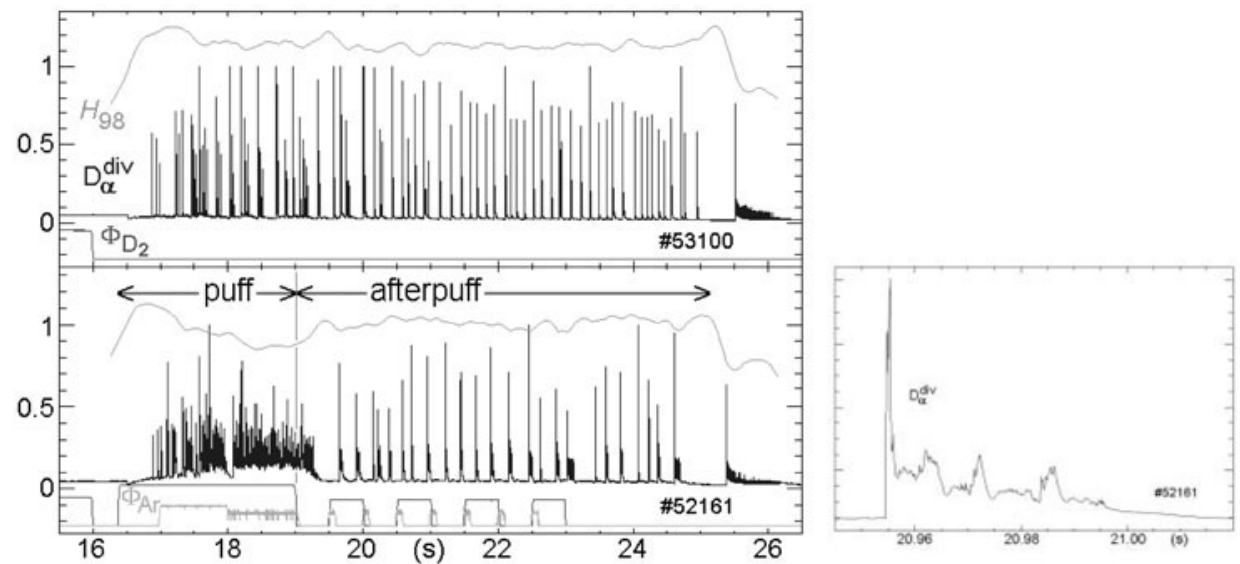

Figure 2. Comparison of VT cases with no gas or seeding (\#53100, top) versus fuelled and argon-seeded (\#52161, bottom), showing normalized confinement, signal proportional to divertor $\mathrm{D} \alpha$ emission, and traces proportional to logarithm of gas input rate (electrons s ${ }^{-1}$ ). 'Puff' (P) and 'afterpuff' (AP) stages are defined, respectively, during and following main fuelling. Inset: expanded view of $\mathrm{D} \alpha$ emission close to $21 \mathrm{~s}$ in \#52161, illustrating the often complex nature of ELM effluxes.

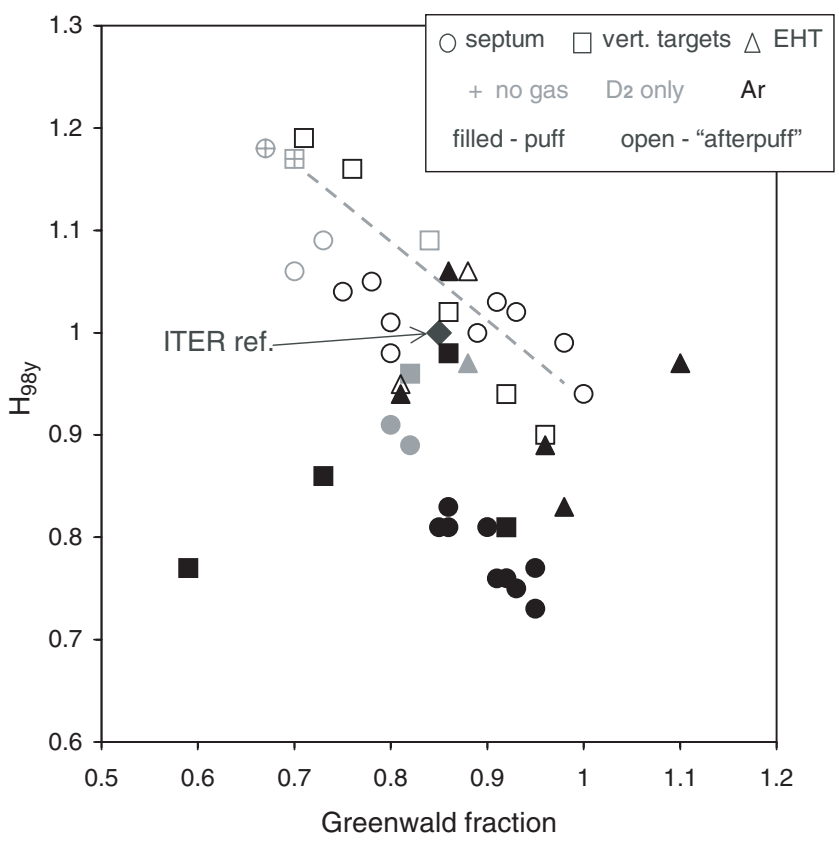

Figure 3. Normalized confinement against normalized density in reference and argon-seeded cases included for all three magnetic configurations. Filled symbols: puff stages; open symbols: afterpuff stages. Seeding allows higher confinement and density to be combined, now encompassing the ITER standard operating point.

while retaining $H_{98} \approx 1$, well above the properties in unseeded or low $\delta$ puff states. In fact, the ITER standard operating point in this diagram is now well covered by JET results. Note that there is a weak tendency for seeded afterpuffs in figure 3 , particularly on the septum, to exhibit greater central peaking of the density, but in terms of a central to volume-averaged ratio from 
LIDAR Thomson scattering it remains always $n_{\mathrm{e} 0} /\left\langle n_{\mathrm{e}}\right\rangle_{\mathrm{V}} \leqslant 1.6$, compared with $\approx 1.3 \rightarrow 1.4$ without argon. Hence, better performance with seeding seems to involve a definite gain in terms of particle confinement and/or refuelling efficiency over the usual H-mode density limit, rather than just profile changes $[1,11,12]$ (see section 4 ). Furthermore, the most dense high $\delta$ seeded puffs actually have even flatter profiles $n_{\mathrm{e} 0} /\left\langle n_{\mathrm{e}}\right\rangle_{\mathrm{V}}<1.3$. These latter plasmas also reach $f_{\text {rad }}>0.6$ for $Z_{\text {eff }} \approx 2$.

\section{ELM frequencies and energy amplitudes}

The vertical targets plasma with gas inputs in figure 2 (\#52161) clearly exemplifies the aptitude during main deuterium puffing at lower $\delta$, noted above, to push ELMs towards Type III features, while depressing confinement. Conversely, it is evident that together with restored confinement, ELM frequency $v_{\mathrm{ELM}}$ instead tends to decrease again during the afterpuff, in fact growing smaller for increasingly potent seeding $[11,12]$. Eventually, $v_{\text {ELM }}$ can be lowered below the level in counterparts without seeding, or even in so-called 'natural' states without any puffing, i.e. their density being determined solely by surface recycling and neutral beam sources. Finally, long ELM-free phases emerge, during which confinement peaks and then declines $[11,12,14]$. This tendency is actually repeated during puff stages at high $\delta$, where argon dosing not only reduces $\nu_{\mathrm{ELM}}$ but promotes a rising $\mathrm{D} \alpha$ level with enhanced irregular activity between ELMs [1,11], reminiscent of the so-called 'mixed Type I + II' fluctuations recently defined [3]. The effect is thus to recover a reciprocal correlation between confinement and ELM frequency [12], i.e. $H_{98}$ is high when $\nu_{\text {ELM }}$ is low, qualitatively similar to the familiar scaling identified by Fishpool for unseeded H-modes in an earlier JET MkIIA divertor design [17]. Recall that the key distinction in seeded plasmas here, though, is that these best conditions of good confinement and slow ELMs are now being achieved at significantly higher radiation and Greenwald fractions $f_{\text {Gwd }} \geqslant 0.9$. Furthermore, core plasma purity is still preserved if sawteeth are maintained, which can be promoted by a small amount $(1 \rightarrow 2 \mathrm{MW})$ of central ICRF heating, as described elsewhere [22]. This technique has been applied in several of the cases included here, for example those at high $\delta$ with modest $Z_{\text {eff }}$ mentioned above.

A likely explanation of these frequency variations is revealed in figure 4 , where $\nu_{\text {ELM }}$ is plotted against power efflux through the plasma edge $P_{\text {edge, estimated as }}$ $P_{\text {edge }} \approx P_{\text {in }}-P_{\text {rad }}^{\text {core }}-\dot{W}$, and radiation from the core is reconstructed from multichord bolometry. As seeding is increased, this latter quantity rises, causing $v_{\mathrm{ELM}}$ to change with falling $P_{\text {edge }}$ for given input power $P_{\text {in }}$, but clearly in quite distinct ways for puff and afterpuff stages. Whereas ELMs follow a definitive Type III scaling $\left(\partial v_{\mathrm{ELM}} / \partial P_{\text {edge }}<0\right)$ in septum and vertical target puffs, they revert to definitive Type I dependence $\left(\partial \nu_{\text {ELM }} / \partial P_{\text {edge }}>0\right)$ in afterpuffs, confirming the nature previously inferred from their better confinement [14]. The latter scaling also prevails for seeded puffs at high $\delta$, which adhere to Type I behaviour throughout. Both of these contexts therefore correspond to a special condition where efflux power can be lessened by augmented radiation, but without breaching the requirements for Type IELMs. This represents a departure from earlier seeding experiments on JET [18], which more closely resembled just the lower $\delta$ puff phases in figure 4. Furthermore, comparing figures 3 and 4 demonstrates that argon injection can combine higher confinement and density with even lower frequency ELMs than can be derived in unseeded or natural plasmas at similar $P_{\text {in }}$ for all three configurations tested. Its relative impact on global properties is seen to be smallest in the vertical targets arrangement, for which pumping is highest. The most effective use of added impurities in terms of global quantities is thus assisted by lower pumping, and in the septum cases up to $\approx \frac{2}{3}$ of augmented radiation is induced around the bulk plasma rather than in the divertor [23], as required to form a mantle. It should be noted that possible involvement 


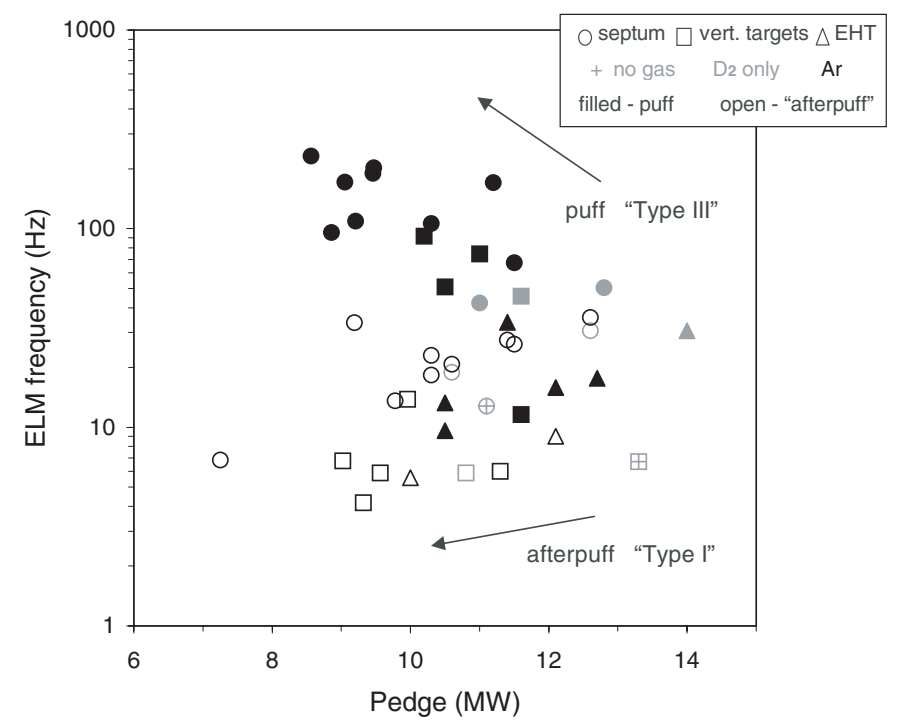

Figure 4. ELM frequency versus estimated power efflux into the plasma edge for the same dataset as in figure 3 (same key; filled symbols: puffs; open symbols: afterpuffs). Strong fuelling with seeding in the puff produces Type III scaling, while gradually more argon dosing in the afterpuff yields Type I scaling.

also of other factors in altering ELM frequency cannot be ruled out, since, as explained in section 4 below, a number of features capable of affecting instability dynamics could not be resolved. However, $v_{\text {ELM }}$ is similar at equal $P_{\text {edge }}$ in septum afterpuffs with and without argon in figure 4 (black/grey open circles, respectively), consistent with radiation being a dominant effect at least for this geometry.

It would conventionally be expected according to a Fishpool relation that time-averaged pedestal pressure and confinement would rise as ELM frequency dropped [17], but that commensurately the fraction of stored energy $(\Delta W / W)$ ejected by each fluctuation would also increase [18]. Energy amplitudes of ELMs can be detected in JET using fast diamagnetic measurement of the stored energy, although only a single $0.8 \mathrm{~s}$ window is presently available, so that fast data cannot be gathered from both puff and afterpuff stages of an individual discharge. Moreover it is common especially with impurity seeding for ELMs to exhibit complex and extremely variable structures, such as illustrated for a single burst mid-way through the vertical targets afterpuff in figure 2. An initial very fast event, typically of $<1 \mathrm{~ms}$ duration, can be followed by relatively much longer, irregular activity, during which energy as well as particles can continue to be expelled. However, since most energy emerges in the leading fast event, and associated power peaks would be the hardest feature to handle in ITER, we have concentrated on amplitudes $(\Delta W / W)$ due only to these dominant components. In order to allow for substantial variability, properties have further been averaged over the largest number of ELMs (up to 29) in each fast window for a subset of the plasmas in figures 3 and 4, hence extending examinations of separate fluctuations in previous studies $[14,15]$. The resulting values of $\langle\Delta W / W\rangle_{\mathrm{t}}$ versus $\left\langle v_{\mathrm{ELM}}\right\rangle_{\mathrm{t}}$ are plotted in figure 5 for lower $\delta$ afterpuffs and both phases at high $\delta$, where instances at highest seeding with long, unsteady ELM-free periods have been excluded. Although there is some scatter, the former septum and vertical targets points are systematically lower with argon $4.3 \% \leqslant\langle\Delta W / W\rangle_{\mathrm{t}} \leqslant 7.6 \%$ than in unseeded references (at lower density), which signify the conventional scaling. In other words, Type I ELMs are reduced in size by impurity 


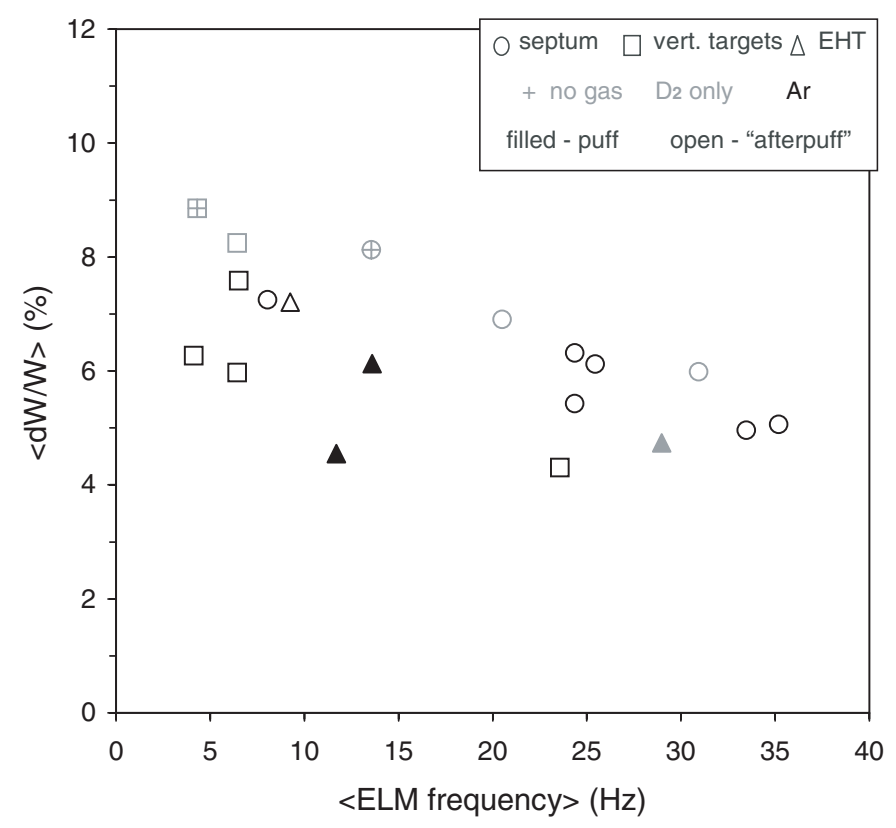

Figure 5. Averaged ELM energy amplitudes versus averaged frequency for a subset of lower $\delta$ afterpuffs and high $\delta$ puffs from figures 3 and 4 . ELMs in seeded afterpuffs are systematically smaller than their unseeded counterparts (at lower density).

injection relative to bursts at comparable frequency in unseeded plasmas. This generalizes a similar finding noted earlier [14], but where features for matching $v_{\mathrm{ELM}}$ were not compared. A separate account of divertor target loads from interpretative modelling of infrared thermography in medium triangularity plasmas has also deduced $\approx 2 \times$ lower peak power densities at the inboard side from ELMs with argon, relative to those of equal frequency without [15]. These observations thus confirm that the usual Fishpool link between Type I ELM frequency and size can be ameliorated with seeding [24], up to $f_{\mathrm{Gwd}} \approx 1$ (figure 3 ) and for a variety of magnetic configurations. They give an indication that as well as aiding core performance, impurity seeding may indeed be able to mitigate power handling in the divertor.

High $\delta$ puffs in figure 5 exhibit no significant change in $\langle\Delta W / W\rangle_{\mathrm{t}}$ but a large decrease in $\left\langle v_{\text {ELM }}\right\rangle_{\mathrm{t}}$ when argon is added. In itself, fewer ELMs per pulse could help to prolong the endurance of intercepted surfaces during ITER standard operation, although ELM power peaks would still remain the dominant factor [25]. One average measurement of the extent to which transient effluxes are moderated may be inferred from the ratio $\varepsilon_{\mathrm{ELM}} \equiv\langle\Delta W\rangle_{\mathrm{t}} \cdot\left\langle\nu_{\mathrm{ELM}}\right\rangle_{\mathrm{t}} /\left\langle P_{\text {in }}\right\rangle_{\mathrm{t}}$ in each unseeded to seeded situation. For septum cases, $\varepsilon_{\mathrm{ELM}}$ is then generally altered from $60 \% \rightarrow 70 \%$ to $50 \% \rightarrow 60 \%$ respectively, mainly because each individual fluctuation contributing to intermittent exhaust is smaller in the latter argon cases (see figure 5). In contrast, for high $\delta$ plasmas, $\varepsilon_{\mathrm{ELM}} \approx 40 \%$ under deuterium puffing falls appreciably to $<20 \%$ at $f_{\mathrm{Gwd}} \approx 1.1$ and $H_{98} \approx 0.97$ adding argon, implying a benefit now of lower $\left\langle v_{\mathrm{ELM}}\right\rangle_{\mathrm{t}}$. Note that radiated power fractions are respectively $\approx 40 \%$ and $>60 \%$ in these experiments, so that in fact amplified inter-ELM energy losses are not immediately suggested in this instance. Vertical targets plasmas have much lower $\varepsilon_{\mathrm{ELM}} \approx 25 \%$ even without seeding, but still reduce to $\approx 10 \%$ at $f_{\text {Gwd }} \approx 0.83$ and $H_{98} \approx 1.0$ with argon, indicating that Type I regimes with less concentrated power efflux are achievable. 


\section{Pedestal properties}

Alteration of ELM behaviour with argon seeding suggests that properties in the pedestal region are being changed, either directly affecting its height or gradients, and/or indirectly changing stability boundaries pertaining to them. In addition, falling $P_{\text {edge }}$ from seeding while the core remains substantially undiluted $[11,12,14]$, especially in cases with central ICRH [22], indicates less power flowing into the pedestal plus perhaps rising radiation within it, again probably modifying its dynamics. Electron pedestal temperature $T_{\mathrm{e}}^{\text {ped }}$ can be derived at moderate field or above in JET from the point of gradient change in edge profiles detected from ECE using a heterodyne radiometer. The accompanying pedestal density $n_{\mathrm{e}}^{\text {ped }}$ is less well resolved, but can generally be approximated [4] for quite flat $\mathrm{H}$-mode profiles from the outer channel of an FIR interferometer viewing along a chord at $3.74 \mathrm{~m}$. An illustration of a combination of these signals for a sequence of septum afterpuffs is shown in figure 6, proceeding from natural to more powerfully argon-seeded states. It is apparent that seeding lowers $T_{\mathrm{e}}^{\text {ped }}$, consistent with radiative cooling of the edge, and that (a) at intermediate argon levels for which ELM frequency remains above the natural rate, this tends to lead to lower electron pedestal pressure peaks just before each fluctuation, i.e. $\left.\hat{p}_{\mathrm{e}}^{\text {ped }}\right|_{\text {Ar }}<\left.\hat{p}_{\mathrm{e}}^{\text {ped }}\right|_{\text {natural }}$. While the ion response has not itself been measured, lower estimated pedestal energy $W^{\text {ped }} \approx 3 p_{\mathrm{e}}^{\text {ped }} V_{\mathrm{p}}$ for plasma volume $V_{\mathrm{p}}$ would consequently imply smaller energy losses in each ELM [15], and so reduced ELM sizes $(\Delta W / W)$ as just described. On the other hand, elevated density $f_{\mathrm{Gwd}}$ with argon dosing is obtained significantly from an increased pedestal density [12], which becomes great enough (b) as more potent seeding lowers $\nu_{\mathrm{ELM}}$ further and $T_{\mathrm{e}}^{\text {ped }}$ also

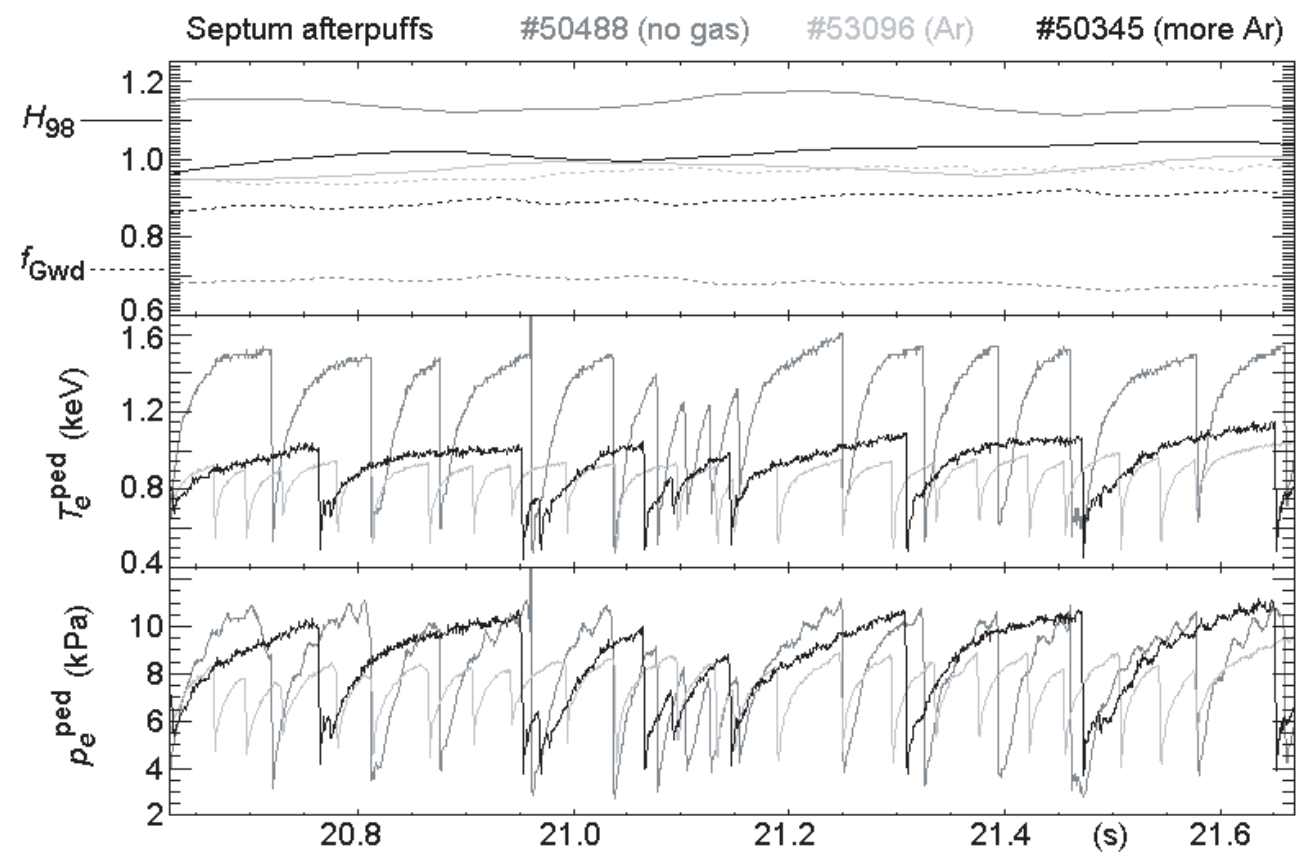

Figure 6. Normalized confinement and density together with electron pedestal temperature and pressure for three septum afterpuffs: no gas ('natural'), then relatively stronger argon seeding. The latter reduces $T_{\mathrm{e}}^{\text {ped }}$, but can eventually slow ELM frequency below the natural rate, allowing $p_{\mathrm{e}}^{\text {ped }}$ to recover the natural level before each fluctuation. 
recovers slightly for the natural peak pedestal pressure to be recovered before each ELM, i.e. $\left.\left.\hat{p}_{\mathrm{e}}^{\text {ped }}\right|_{\text {stronger Ar }} \approx \hat{p}_{\mathrm{e}}^{\text {ped }}\right|_{\text {natural }}$, even for $\left.v_{\mathrm{ELM}}\right|_{\text {stronger Ar }}<\left.v_{\mathrm{ELM}}\right|_{\text {natural }}$, as in figure 6 . Recall that ELM sizes are still slightly smaller (or comparable within uncertainities) in the seeded relative to the natural case here, despite their lower frequency (cf figure 5). However, ion data, plasma rotation, and especially edge current density would all be needed quantitatively to test whether MHD stability boundaries in the pedestal had really been altered by argon. Note that pedestal width/pressure gradient is also not resolved, and edge profiles in an earlier study [14] were actually determined at a low diagnostic sampling rate, such that it is uncertain whether they coincided with the upper limits of ELM cycles. Nevertheless the rise to $\left.\hat{p}_{\mathrm{e}}^{\text {ped }}\right|_{\text {stronger Ar }}$ is seen in figure 6 to be governed largely by the rate of edge refuelling rather than reheating, since $T_{\mathrm{e}}^{\text {ped }}$ tends quickly to saturate while $n_{\mathrm{e}}^{\text {ped }}$ evidently continues to grow (very well seen for pulse \#50345 between 20.80 and 20.95 s). Recycling therefore seems to play a prominent role in ELM occurrence under these radiating mantle conditions. At least in part, ELM frequencies are evidently lowered by impurities slowing pedestal recovery processes between fluctuations [24].

A similar pattern of ELM and pedestal effects with argon is repeated in the vertical targets afterpuffs, particularly saturation of $T_{\mathrm{e}}^{\text {ped }}$ while $n_{\mathrm{e}}^{\text {ped }}$ steadily increases up to each ELM, despite stronger pumping. Few equivalent data have been obtained yet in seeded puffs at high $\delta$, but these will be addressed in the next experimental campaign on JET. A summary of electron pedestal values immediately prior to an ELM in a subset of plasmas from figure 5 is plotted in figure 7. The progression for septum afterpuffs outlined above is clearly reiterated, while the largest relative changes are actually found in vertical targets configuration, which it should be recalled exhibits the smallest variation in $v_{\text {ELM }}$ with argon injection. This again suggests that movements in MHD stability boundaries are possibly involved as well. Another striking

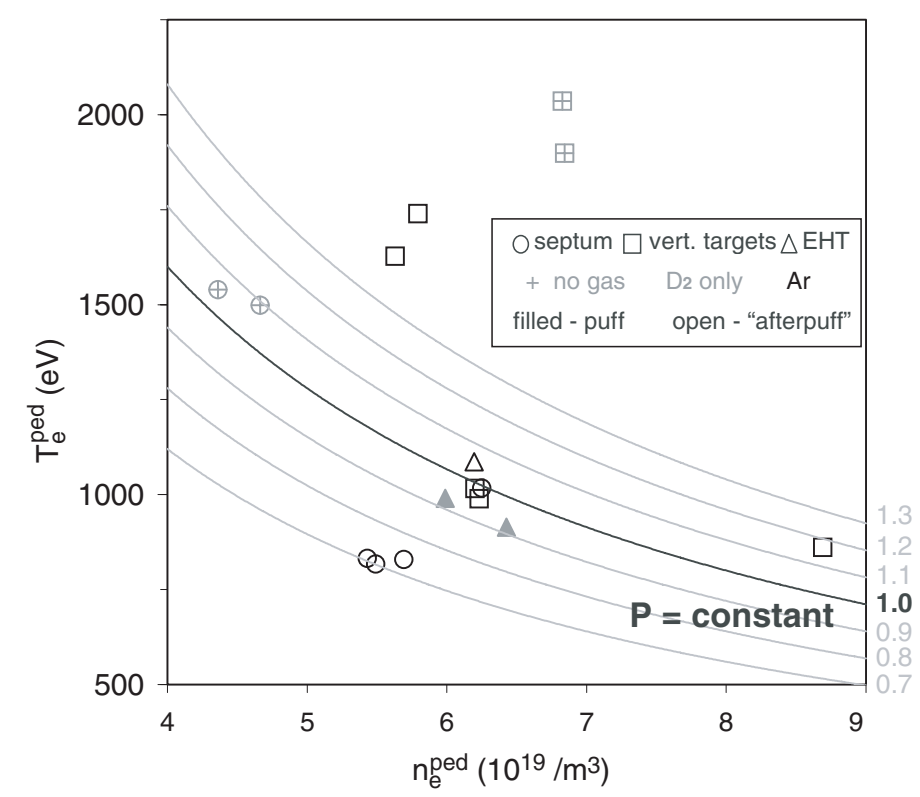

Figure 7. Electron pedestal properties just prior to an ELM in a subset of plasmas from figure 5. In septum afterpuffs the natural pedestal pressure is eventually recovered as argon seeding reduces ELM frequency below the natural rate. 
feature is that very similar absolute pedestal properties are recovered in some examples of each geometry including argon, i.e. seeding may lessen the configurational sensitivity of the pedestal.

Estimated pedestal energy $W^{\text {ped }}$ and its fraction of total content $\left(W^{\text {ped }} / W\right)$ are thus lowered in seeded afterpuffs with relatively more frequent ELMs, which in fact also tend to reach highest plasma densities. Septum cases, for example, progress from $\left(W^{\text {ped }} / W\right) \approx 44 \%$ at $f_{\mathrm{Gwd}} \approx 0.69, H_{98} \approx 1.1$ and $\nu_{\mathrm{ELM}} \approx 15 \mathrm{~Hz}$ in natural H-mode, to $\left(W^{\text {ped }} / W\right) \approx 33 \%$ at $f_{\mathrm{Gwd}} \approx 0.96, H_{98} \approx 0.92, \nu_{\mathrm{ELM}} \approx 20 \mathrm{~Hz}$ with moderate $\operatorname{argon}$, then retrieve $\left(W^{\text {ped }} / W\right) \approx$ $47 \%$ at $f_{\mathrm{Gwd}} \approx 0.87, H_{98} \approx 1.0, v_{\mathrm{ELM}} \approx 7 \mathrm{~Hz}$ under strongest argon radiation. Analyses of local transport with the TRANSP code have previously indicated reductions in effective thermal diffusivities $\chi_{\text {eff }}$, combining electron and ion channels, between such unseeded to seeded afterpuffs [20], as would be expected for an RI-like effect resembling that first observed in TEXTOR-94 [9]. Further calculations for vertical targets plasmas find that $\chi_{\text {eff }}$ is similar in afterpuffs with and without argon seeding, and lower than in puff phases. Energy confinement, in other words, is equally improved over its value during main fuelling in each afterpuff, but recall that seeding preserves this good performance above the H-mode density limit otherwise intervening [4,12]. At lower triangularity, changes in $H_{98}$ like those listed therefore tend to remain in close proportion to $\left(W^{\text {ped }} / W\right.$ ), which in turn is inversely related to $\nu_{\mathrm{ELM}}$, qualitatively in accordance still with the Fishpool interpretation for unseeded plasmas [17], i.e. $\left\langle W^{\text {ped }}\right\rangle_{t}$ decreases for greater $\nu_{\mathrm{ELM}}$. Simultaneously, an unequivocal effect of impurity injection is exerted on confinement of particles and/or refuelling efficiency $[1,11,12]$, as already inferred above, and TRANSP results for vertical targets cases in figure 8 indeed derive significantly lower electron diffusivity $D_{\perp}$ in a seeded afterpuff than in a natural state. This occurs despite respective core density profiles being very similar. The more integrated afterpuffs displaying superior performance together with moderated ELM amplitudes or frequencies hence involve

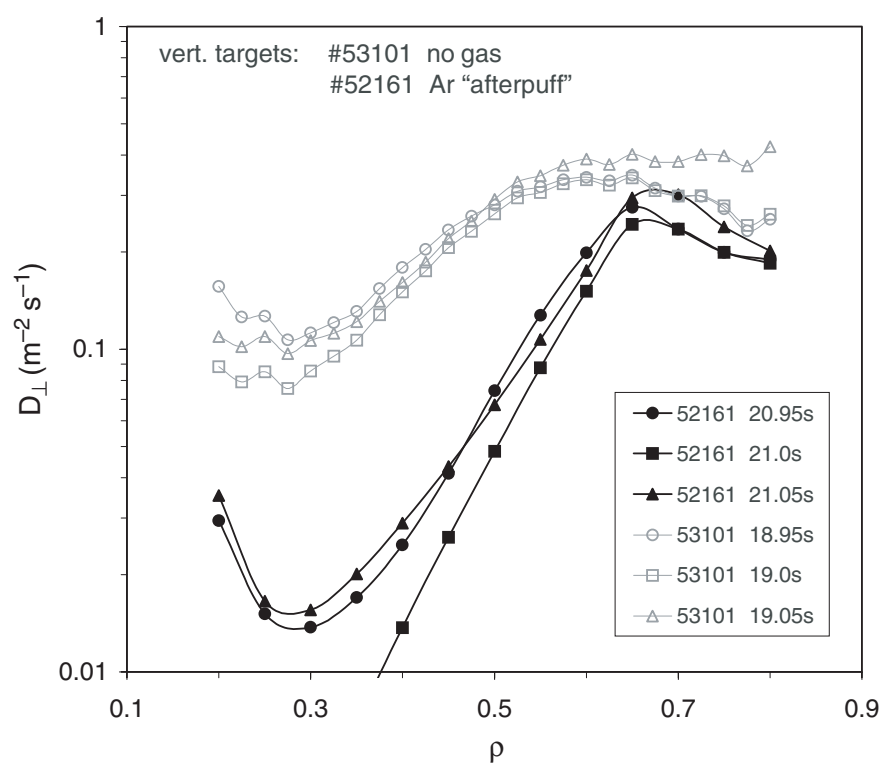

Figure 8. Estimated radial profiles of electron diffusivity $D_{\perp}$ calculated with the TRANSP code during vertical targets plasmas as in figure 2. Particle transport is reduced in the seeded afterpuff (\#52161), though respective core density profiles are very similar. 


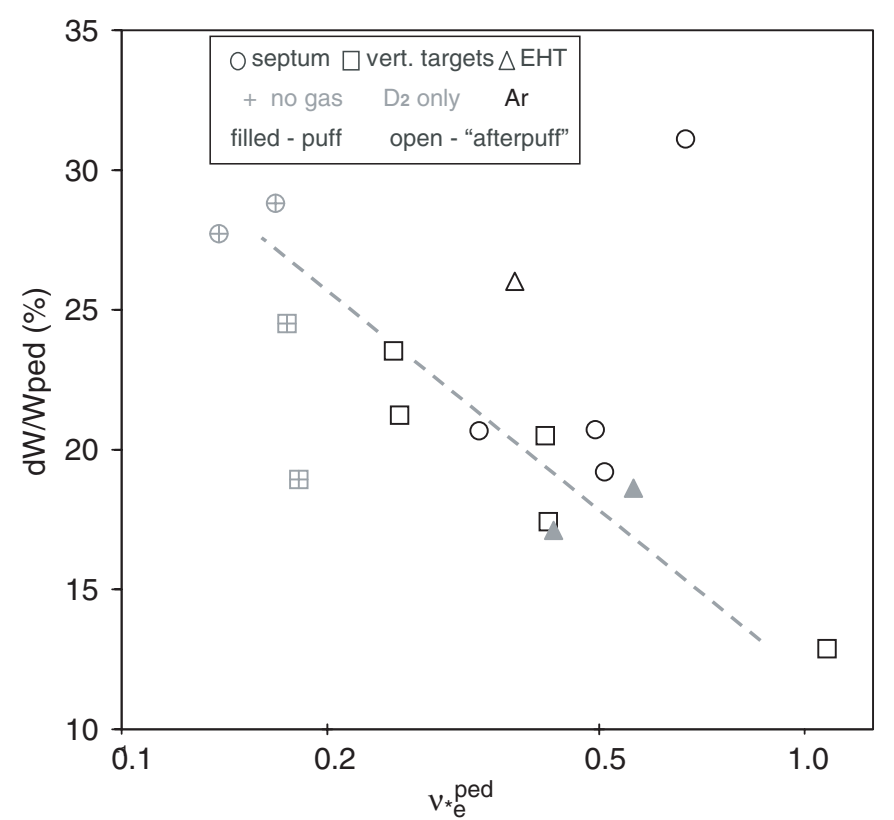

Figure 9. ELM size normalized to estimated pedestal energy versus electron collisionality in the pedestal just before a fluctuation. Same dataset and key as in figure 7. Argon seeding reduces ELM losses, but does not disturb the reciprocal correlation $\sim \ln \left\{\left(v_{* \mathrm{e}}^{\text {ped }}\right)^{-0.1}\right\}$ prevailing in all three configurations used.

two separate benefits of seeding: lower transport especially of particles in the core; plus tempered ELM fluctuations mainly through action in the edge. Note that the mechanism of transport suppression by impurities remains under study, but it has been shown before that ion temperature gradient (ITG) mode turbulence can be weakened directly by their higher charge, even in the absence of pronounced density peaking [26].

While variations in ELM frequency may depend on complex re-excursions of stability boundaries, current understanding of Type I ELM sizes is that they are determined by pedestal properties through a reciprocal correlation [24] between $\left(\Delta W / W^{\text {ped }}\right)$ and local collisionality $\nu_{* \mathrm{e}}^{\mathrm{ped}}$, where $\nu_{* \mathrm{e}} \propto Z_{\mathrm{eff}}\left(n_{\mathrm{e}} / T_{\mathrm{e}}^{2}\right)$. This dependence is examined for the foregoing dataset in figure 9 , assuming radially constant $Z_{\text {eff }}$ values. The latter approximation may be partly responsible for some evident scatter, but a qualitatively similar result $\left(\Delta W / W^{\text {ped }}\right) \sim$ $\ln \left\{\left(v_{* e}^{\text {ped }}\right)^{-0.1}\right\}$ is generally preserved when injecting argon, in all three configurations. The essential physics of Type I ELM instabilities, yet to be resolved [24], does therefore seem to be unaffected by impurity seeding. In contrast, $\left(\Delta W / W^{\text {ped }}\right)$ exhibits no clear correlation versus $\nu_{\mathrm{ELM}}$ over these cases, emphasizing again the departure from a familiar Fishpool connection $[17,18]$ between ELM frequency and size that seeding can induce. The universal form of figure 9 also underlines that ELM sizes are reduced as impurity radiation cools [15] $T_{\mathrm{e}}^{\mathrm{ped}}$ and raises pedestal collisionality, i.e. mainly due to seeding effects at the edge, as commented above, and compatible with creation of a radiating mantle. Together these features consequently imply that $(\Delta W / W)=\left(\Delta W / W^{\text {ped }}\right) \cdot\left(W^{\text {ped }} / W\right)$ in figure 5 declines with $\nu_{\text {ELM }}$ primarily as ( $W^{\text {ped }} / W$ ) (and $H_{98}$ slightly) drops, while it is smaller with seeding at a given ELM frequency as $\left(\Delta W / W^{\text {ped }}\right)$ falls at higher edge collisionality. Radiation from added impurities can thus cool and slow recovery of the pedestal, yielding beneficial reductions 
of both Type I ELM sizes and frequency, integrated with enhanced plasma confinement in the core.

\section{Summary and discussion}

Argon-seeded H-mode experiments on JET have demonstrated that good confinement and density close to the Greenwald level can be combined in quasi-stationary conditions at lower plasma triangularity, overcoming the former divertor H-mode density limit. An optimized fuelling and radiation strategy is employed, involving a preparatory puff and a high performance afterpuff stage. Similar benefits are found with argon injection into the main puffing phase at high triangularity, but so far these have not been developed into steady states. Results now encompassing the ITER standard operating point $H_{98} \approx 1, f_{\mathrm{Gwd}} \approx 0.85$ are thus achieved by retaining Type I ELMs at higher density, through improved particle confinement and/or refuelling efficiency. Energy confinement in the core is preserved or may even be improved as well $[13,20]$.

Sets of plasmas surveyed in low $\delta$ pumped-limiter-like septum, and medium $\delta$ divertor, configurations show that seeding simultaneously reduces the average energy amplitudes $\langle\Delta W / W\rangle_{\mathrm{t}}$ of explicitly Type I ELMs below the sizes predicted for comparable $H_{98}$ and frequency (but lower density) by conventional scaling $[17,18]$. This generalizes a related finding in earlier studies $[14,15]$. Decreases of up to $\approx 30 \%$ are produced by radiative cooling of the electron pedestal temperature $T_{\mathrm{e}}^{\text {ped }}$. Equivalent divertor plasmas at high $\delta$ appear not to exhibit such reductions of $\langle\Delta W / W\rangle_{\mathrm{t}}$, but in all cases average Type I ELM frequency $\left\langle v_{\mathrm{ELM}}\right\rangle_{\mathrm{t}}$ is alternatively lessened by argon owing to lowering of power efflux through the edge, which in turn slows inter-ELM pedestal recovery processes. For higher radiation fractions from seeding at lower $\delta$, the latter appears to be dominated by refuelling of the pedestal density rather than edge reheating, so that recycling must then play a role in ELM cycles. Together these factors lead to a general, significant moderation of average power efflux in ELMs, with lowest examples so far obtained yielding $\langle\Delta W\rangle_{\mathrm{t}} \cdot\left\langle v_{\mathrm{ELM}}\right\rangle_{\mathrm{t}} /\left\langle P_{\mathrm{in}}\right\rangle_{\mathrm{t}} \approx 25 \%$ and $10 \%$ respectively in septum and medium $\delta$ divertor arrangements, both still for $H_{98} \approx 1$.

Higher plasma density with impurity injection is derived appreciably from a higher pedestal density $n_{\mathrm{e}}^{\text {ped }}$. While cooling of $T_{\mathrm{e}}^{\text {ped }}$ at lower $\delta$ can therefore lower local peak pressure $\hat{p}_{\mathrm{e}}^{\text {ped }}$ immediately before an ELM, in septum geometry elevation of $n_{\mathrm{e}}^{\text {ped }}$ eventually compensates, so that at high enough radiation fractions pedestal pressure and its related energy content recover to unseeded levels. This contributes to improved performance eventually with lower frequency ELMs than in natural states on the septum, compared with which argon seeding decreases their frequency by a factor of $\approx 2$ for very similar size. At the same time, the underlying physics of Type I instabilities as described in terms of pedestal collisionality [24] seems always to be unaltered by seeding. Although matching pedestal data for high $\delta$ cases have yet to be obtained, better integrated $\mathrm{H}$-modes generally benefit from two separate effects of seeding, viz lower transport particularly of particles in the core, plus ELM abatement due to a cooler edge.

Dosing H-mode plasmas with argon can thus assist not only global performance, but also mitigation of both steady and transient power effluxes, through mantle radiation, plus reductions of Type I ELM sizes and/or frequency. Sensitivity of the effects to a number of detailed factors, including deuterium and argon inputs, sawteeth to protect core purity, pumping to maximize edge radiation, and recycling, also suggests that further optimization may yet be possible. In particular, promising results are emerging in latest JET experiments incorporating active feedback control of gas puff rates [27], recalling methods successfully applied in the 
original seeding studies on TEXTOR-94 [9] and ASDEX-U [10]. These techniques will next be extended to high $\delta$ configuration, which most closely resembles the shapes expected to be used in ITER. Recent modelling [28] has suggested in addition that the benefits of impurity radiation both on steady and sudden heat exhaust may even be improved in larger devices using heavier species, namely krypton rather than argon. There are hence clear indications that seeding, for example with krypton, could access high density H-modes with enhanced average radiation plus moderated Type I ELM loads in ITER, as required to realize a burning plasma regime together with longer component lifetimes, especially in its divertor. A fully integrated scenario for its standard $Q=10$ operation might well then be obtained.

\section{Acknowledgments}

This work has been conducted under the European Fusion Development Agreement and was partly funded by Euratom and the UK Department of Trade \& Industry.

\section{References}

[1] Ongena J et al 2001 Plasma Phys. Control. Fusion 43 A11

[2] Kubo H et al 2002 Phys. Plasmas 92127

[3] Saibene G et al 2002 Plasma Phys. Control. Fusion 441769

[4] Saibene G et al 1999 Nucl. Fusion 391133

[5] Loarte A et al 2000 Proc. 18th IAEA Fusion Energy Conf. (Sorrento, October 2000) (Vienna: IAEA) ITERP/11(R)

[6] Ozeki T et al 1990 Nucl. Fusion 301425

[7] Kamada Y et al 1996 Plasma Phys. Control. Fusion 381387

[8] Stober J et al 2001 Nucl. Fusion 411123

[9] Messiaen A M et al 1994 Nucl. Fusion 34825

[10] Kallenbach A et al 1995 Nucl. Fusion 351231

[11] Dumortier P et al 2002 Plasma Phys. Control. Fusion 441845

[12] Maddison G P et al 2003 Nucl. Fusion 4349

[13] Strachan J D et al 2000 Plasma Phys. Control. Fusion 42 A81

[14] Jachmich S et al 2002 Plasma Phys. Control. Fusion 441879

[15] Rapp J et al 2002 Proc. 19th IAEA Fusion Energy Conf. (Lyon, October 2002) Nucl. Fusion EX/P1-09 submitted

[16] Monier-Garbet P et al 2003 30th EPS Conf. on Controlled Fusion and Plasma Physics (St Petersburg, July 2003)

[17] Fishpool G M 1998 Nucl. Fusion 381373

[18] Matthews G F et al 1999 Nucl. Fusion 3919

[19] Horton L D et al 1999 Proc. 26th EPS Conf. on Controlled Fusion and Plasma Physics (Maastricht, June 1999) vol 23J (ECA) p 193, CD-ROM file P1.021

[20] Ongena J et al 2001 Phys. Plasmas 82188

[21] Kallenbach A et al 2002 Nucl. Fusion 421184

[22] Nave M F F et al 2003 Nucl. Fusion at press

[23] Hillis D L et al 2001 Proc. 43rd Meeting DPP of APS (Long Beach, October 2001)

[24] A Loarte et al 2002 Plasma Phys. Control. Fusion 441815

[25] Federici G et al 2003 J. Nucl. Mat. 11 313-16

[26] Tokar M Z et al 2002 Plasma Phys. Control. Fusion 441903

[27] Dumortier P et al 30th EPS Conf. on Controlled Fusion and Plasma Physics (St Petersburg, July 2003)

[28] Monier-Garbet P et al 2002 Proc. 44th Meeting DPP of APS (Orlando, November 2002) 